\title{
Nonlinear diffusion control of spatiotemporal chaos in the complex Ginzburg-Landau equation
}

\author{
Raúl Montagne* \\ Departament de Física, Universitat de les Illes Balears, E-07071 Palma de Mallorca, Spain \\ Pere Colet \\ Instituto Mediterráneo de Estudios Avanzados, IMEDEA (CSIC-UIB), E-07071 Palma de Mallorca, Spain
}

(Received 11 December 1996; revised manuscript received 3 July 1997)

\begin{abstract}
The role of nonlinear diffusion terms in the stability of periodic solutions in the regime of spatiotemporal chaos is studied. The stabilization of unstable plane waves in the complex Ginzburg-Landau equation in weakly chaotic regimes such as phase turbulence and spatiotemporal intermittency or in strong chaotic ones such as defect turbulence is demonstrated. [S1063-651X(97)07410-2]

PACS number(s): 05.45.+b, 47.54.+r, 82.40.Bj
\end{abstract}

\section{INTRODUCTION}

Under nonequilibrium conditions, a spatially extended system often undergoes a transition from a uniform state to a state with spatial variation, usually referred to as a pattern. Their formation is generally associated with nonlinear effects, which in may cases can lead to qualitatively different phenomena such as spatiotemporal chaos [1] (STC). Loosely, the term spatiotemporal chaos is commonly accepted to refer to a deterministic system that has irregular variation and is unpredictable in detail both in space and in time. There are known examples of experimental systems, well characterized and precisely controlled [2,1], that show such behavior. In most cases STC can be described within the context of weakly nonlinear theories since these states arise in the proximity of a threshold. These theories are well developed in the form of so-called complex GinzburgLandau equations (CGLEs) [3]. The CGLE is a prototypical equation for a complex field $A$ that exhibits STC. It accounts for the slow modulations in space and time of the oscillatory state in a physical system that undergoes a Hopf bifurcation [4]. The CGLE shows several types of STC [5] that have been extensively studied [6-10].

The control of the chaotic behavior of dynamical systems with few degrees of freedom has been successively tested in a number of systems [11]. The idea behind the control of chaos is to modify the dynamics of the system in such a way that a previously unstable state is now stable. Ideally, only the stability is modified, not the state itself (i.e., if that state was a fixed point or periodic orbit of the original system it is still a fixed point or periodic orbit of the modified system). The control of spatiotemporal chaos is a more complicated problem and so there is a wide variety of methods intended to control such chaotic behavior. There have been several attempts to achieve such control in the CGLE. For example, Aranson et al. [12] stabilized a structurally unstable topological defect, whose analytical expression is known, by adding an extra term in the CGLE. The defect acts as a source of traveling waves, which sweep all the other fluctuations to the

\footnotetext{
*Present address: Instituto de Física, Facultad de Ciencias, T. Narvaja 1674, Código Postal 11200, Montevideo, Uruguay.
}

system boundary. Stabilization of a plane wave extended through the entire system has been achieved by adding timedelayed feedback terms to the CGLE. The feedback can be either local [13] (at each spatial point, the field at the same point at previous times is fed back) or global $[14,15]$ (at each spatial point a term proportional to the integral of the field over the spatial variable is fed back). In both cases, the added terms vanish for the stabilized plane-wave solution, so it is possible to stabilize precisely the same plane waves that are unstable in the original CGLE. However, the added global feedback terms do not preserve the phase invariance of the original CGLE.

Feedback is the most often used approach for chaos control in spatially extended systems. It has been applied to a nonlinear drift-wave equation driven by a sinusoidal wave [16] and, in conjunction with a spatial filter, it has also been applied to stabilize rolls and hexagonal structures in a model for a transversally extended three-level laser [17] and to control filamentation in a model for wide aperture semiconductor lasers based on the Swift-Hohenberg equation [18].

In this paper we explore a different way to stabilize unstable periodic solutions based not on feedback terms but on nonlinear diffusion effects. Specifically, we show that stabilization of unstable plane-wave solutions of the CGLE in the region of STC can be achieved adding a nonlinear diffusion (or diffraction) term. The added term preserves the intrinsic phase invariance of the CGLE equation and vanishes when the stabilizing effect is achieved, so any plane-wave solution of the original CGLE is also solution of the modified equation. Nonlinear diffraction effects are present in optical systems where the Fresnel number is intensity dependent or in systems where the refraction index is intensity dependent, such as in photorefractive materials.

In Sec. II we briefly describe the parameter regions for which different chaotic behaviors have been found for the CGLE and we introduce the modified equation. Section III is devoted to the linear stability analysis of the plane-wave solutions. We calculate for which parameter values the added term is able to stabilize plane waves in the STC regions of the CGLE. In Sec. IV we show, by integrating the equations numerically, that the stability of the plane waves when finitesize perturbations are applied is in excellent agreement with the analytical prediction of the linear stability analysis. Fi- 


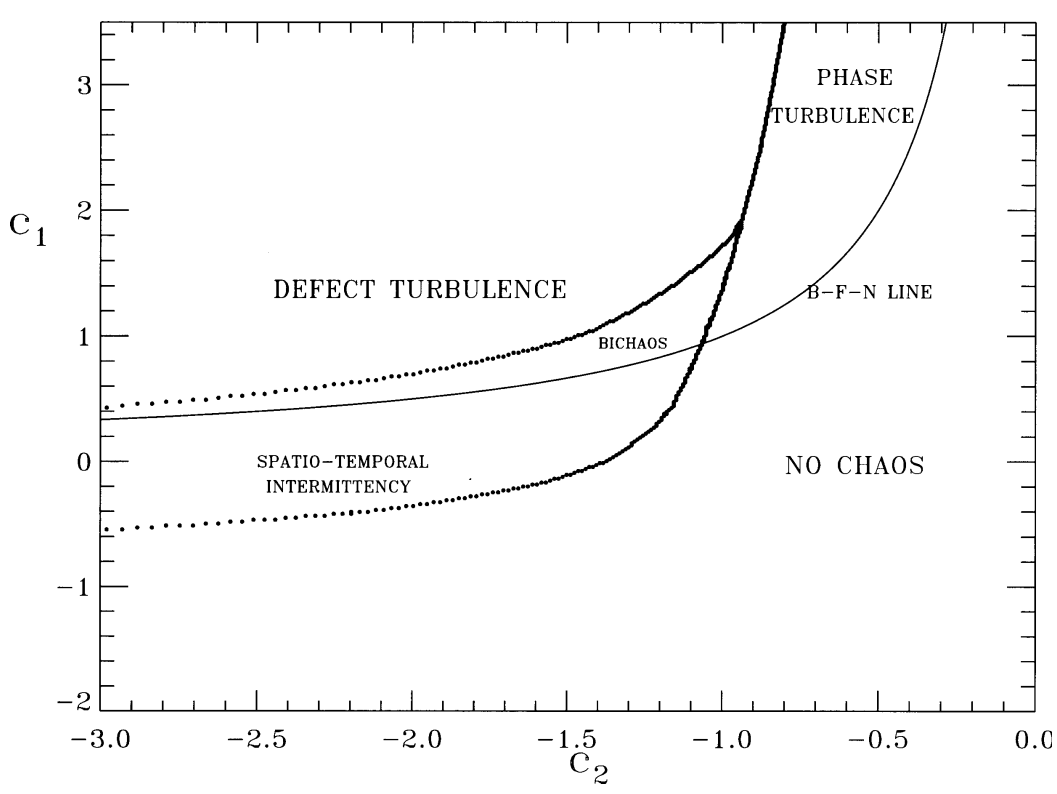

FIG. 1. Regions of the parameter space $c_{1}-c_{2}$ for which the $d=1$ CGLE displays different kinds of regular and chaotic behavior. The analytically obtained line, the Benjamin-Feir-Newell line (BFN line), is also shown. The quantities plotted in all the figures of this paper are dimensionless.

nally, we give some concluding remarks in Sec. V.

\section{MODEL}

The one-dimensional CGLE $[3,4,19,20]$ for a complex field $A(x, t)$ describes the slow dynamics of spatially extended systems close to a Hopf bifurcation,

$$
\partial_{t} A=A+\left(1+i c_{1}\right) \partial_{x}^{2} A-\left(1+i c_{2}\right)|A|^{2} A .
$$

We will assume periodic boundary conditions throughout the paper. This equation admits plane-wave solutions of the form

$$
A_{\mathrm{PW}}(x, t)=A_{0} e^{i(k x-\omega t)},
$$

with amplitude $A_{0}=\sqrt{1-k^{2}}, \quad|k|<1$, and frequency $\omega=c_{2}+\left(c_{1}-c_{2}\right) k^{2}$.

For $1+c_{1} c_{2}>0$ plane-wave solutions are linearly stable for wave numbers smaller than a limit value $|k| \leqslant k_{E}$. For $|k|>k_{E}$, plane waves are unstable to phase perturbations (Eckhaus instability [21]). The limit value $k_{E}$ is given by

$$
k_{E}^{2}=\frac{1+c_{1} c_{2}}{3+c_{1} c_{2}+2 c_{2}^{2}} .
$$

The stability range vanishes at $1+c_{1} c_{2}=0$ [the BenjaminFeir-Newell (BFN) line] and no stable plane-wave solution exists for $1+c_{1} c_{2}<0$.

Numerical work for $L$ (length of the system) large $[5-10,22]$ has identified regions of the parameter space displaying different kinds of regular and spatiotemporal chaotic behavior, leading to a "phase diagram" for the CGLE in the plane $c_{1}-c_{2}$. The five different regions, each leading to a different asymptotic phase, are shown in Fig. 1 as functions of the parameters $c_{1}$ and $c_{2}$. Two of these regions are in the BFN stable zone and the other three in the BFN unstable one. The "no chaos" region in the BFN stable zone is a large region where the evolution ends in a plane wave with a wave number $|k| \leqslant k_{E}$ for almost all the initial conditions. Also in the BFN stable zone there is the spatiotemporal intermittency region [7]. Despite the fact that there exist stable plane waves, the evolution from random initial conditions is not attracted to them but to a chaotic attractor in which typical configurations of the field $A$ consist of patches of plane waves interrupted by turbulent bursts. The modulus of $A$ in such bursts typically touches zero quite often. Above BFN line, the evolution ends in a spatiotemporal chaos for almost every initial condition. The defect turbulence region is a strongly disordered region in which the modulus of $A$ has a finite density of space-time zeros [6,7]. The phase turbulence $[5,6,8,23-25]$ region is a weakly disordered one in which $|A(x, t)|$ remains away from zero. Nevertheless, under a particular type of initial condition it is possible to end in a ordered state [10,22]. Finally, the bichaos region is such that, depending on the particular initial condition, the system ends on attractors similar to the ones in regions of phase turbulence or defect turbulence or in a new attractor in which the configurations of $A$ consists of patches of phase and defect turbulence. A detailed description can be found in [5].

We consider a modification of CGLE in such a way that the plane-wave stability region is increased. A way to do this is by changing a parameter of the system dynamically and proportionally to the deviation of the system from the state to be stabilized. We will show that stabilization of plane waves can be achieved by replacing the coefficient $c_{1}$ by $c_{1}+\gamma\left(|A|^{2} /\left|A_{\mathrm{PW}}\right|^{2}-1\right)$, where $\gamma$ is a constant and $\left|A_{\mathrm{PW}}\right|$ is the modulus of the plane wave to be stabilized $\left|A_{\mathrm{PW}}\right|=A$. Notice that as the added term $\epsilon\left(|A|^{2} /\left|A_{\mathrm{PW}}\right|^{2}-1\right)$ vanishes identically for $A=A_{\mathrm{PW}}$, any plane wave $A_{\mathrm{PW}}$ that is a solution of Eq. (2.1) is also a solution of the modified equation. We are not changing the solution, but we will change its stability. The added term also preserves the phase invariance of the solution of the original CGLE, $A \rightarrow A e^{i \psi}$, with $\psi$ being an arbitrary phase. The modified CGLE is then explicitly given by 


$$
\begin{aligned}
\partial_{t} A= & A+\left[1+i c_{1}+i \gamma\left(|A|^{2} /\left|A_{\mathrm{PW}}\right|^{2}-1\right)\right] \partial_{x}^{2} A \\
& -\left(1+i c_{2}\right)|A|^{2} A .
\end{aligned}
$$

From another point of view, Eq. (2.4) can be rewritten as

$$
\partial_{t} A=A+\left[1+i \widetilde{c_{1}}+i c_{\mathrm{NL}}|A|^{2}\right] \partial_{x}^{2} A-\left(1+i c_{2}\right)|A|^{2} A,
$$

with $\widetilde{c_{1}}=c_{1}-\gamma$ and $c_{\mathrm{NL}}=\gamma /\left|A_{\mathrm{PW}}\right|^{2}$. In this way, the stabilizing added term can be seen explicitly as a nonlinear diffusive term in the CGLE.

\section{STABILITY ANALYSIS}

We study the stability of the plane-wave solutions (2.2) of Eq. (2.4) using a standard linearization procedure. Consider the time evolution of small perturbations in the amplitude and phase

$$
A(x, t)=\left[A_{0}+\epsilon r(x, t)\right] e^{i[k x-\omega t+\epsilon \phi(x, t)]},
$$

where $r(x, t)$ and $\phi(x, t)$ are real perturbations in the amplitude and phase, respectively, and $\epsilon$ is a formal parameter to keep track of small numbers.

Substituting Eq. (3.1) into Eq. (2.4) yields to a polynomial in $\epsilon$ up to order $\epsilon^{5}$. The terms of order $\epsilon^{0}$ vanish identically. The first-order terms yield the linearized equations for the perturbations

$$
\begin{aligned}
\partial_{t} r= & 2 A_{0}^{2} r-2 A_{0} k \partial_{x} \phi-2 c_{1} k \partial_{x} r-c_{1} A_{0} \partial_{x}^{2} \phi+\partial_{x}^{2} r \\
\partial_{t} \phi= & -2 c_{2} A_{0} r+2 \gamma \frac{k^{2}}{A_{0}} r-2 c_{1} k \partial_{x} \phi+2 \frac{k}{A_{0}} \partial_{x} r+\partial_{x}^{2} \phi \\
& +\frac{c_{1}}{A_{0}} \partial_{x}^{2} r .
\end{aligned}
$$

We consider solutions of Eqs. (3.2) and (3.3) proportional to $e^{\eta t+i q x}$, where for periodic boundary conditions $q$ is real whereas $\eta$ is in general a complex quantity. By substituting into Eqs. (3.2) and (3.3), we obtain the dispersion relation

$$
\left|\begin{array}{cc}
\eta+2 A_{0}^{2}+q^{2}+2 i c_{1} q k & 2 i q k-c_{1} q^{2} \\
c_{1} q^{2}+2 c_{2} A_{0}^{2}-2 i q k+2 \gamma k^{2} & \eta+q^{2}+2 i c_{1} q k
\end{array}\right|=0 .
$$

The solutions of Eq. (3.4) are

$$
\eta=-\left(A_{0}^{2}+q^{2}+2 i c_{1} q k\right) \pm \sqrt{u+i v},
$$

where $u$ and $v$ are polynomials

$$
\begin{gathered}
u=A_{0}^{4}+4 q^{2} k^{2}-2 c_{1} c_{2} A_{0}^{2} q^{2}-c_{1}^{2} q^{4}-2 \gamma c_{1} q^{2} k^{2}, \\
v=4 q k\left(c_{1} q^{2}+c_{2} A_{0}^{2}+\gamma k^{2}\right) .
\end{gathered}
$$

The real part of $\eta$ indicates the growth rate of the perturbations

$$
\operatorname{Re}(\eta)=-A_{0}^{2}-q^{2} \pm \sqrt{\frac{u+\sqrt{u^{2}+v^{2}}}{2}} .
$$

We have two different branches $[4,26,27]$ that are usually called "amplitude" and "phase modes" due to the fact that for a real Ginzburg-Landau equation the eigenvalues are related specifically to amplitude and phase perturbations. Although this is not the case for the CGLE, the names are still used.

The amplitude modes correspond to the negative sign of the square root in Eqs. (3.8). For any value of $c_{1}, c_{2}$, and $k$, the growth rate $\operatorname{Re}(\eta)$ as function of the perturbation wavelength $q$ is always negative and takes the value $\operatorname{Re}(\eta)=-2 A_{0}^{2}$ at $q=0$. The added $\gamma$ term modifies slightly the value of $\operatorname{Re}(\eta)$, but it never changes its sign, so these perturbations are always damped.

The phase modes are associated with the positive sign of the square root in Eq. (3.8). The growth rate vanishes identically at $q=0$ for any value of the parameters $c_{1}, c_{2}$, and $k$, so all the plane-wave solutions are marginally stable. The origin of this neutral stability is the phase invariance $A \rightarrow A e^{i \psi}$ of the solutions of Eqs. (2.1) and (2.4). For $q$ very large, the growth rate is negative and behaves as $-q^{2}$, so short-wavelength perturbations are always damped. However, long-wavelength perturbations can grow, destabilizing the original plane-wave solution; to see this we expand Eq. (3.8) for small $q$

$$
\operatorname{Re}(\eta)=D q^{2}+O\left(q^{4}\right),
$$

where

$$
\begin{aligned}
D= & -1-c_{1} c_{2}+2\left(1+c_{2}^{2}\right) \frac{k^{2}}{A_{0}^{2}}+\gamma\left(-\frac{c_{1} k^{2}}{A_{0}^{2}}+4 \frac{c_{2} k^{4}}{A_{0}^{4}}\right) \\
& +2 \gamma^{2} \frac{k^{6}}{A_{0}^{6}} .
\end{aligned}
$$

If this coefficient is positive, there is a range of longwavelength perturbations that grow. The condition $D<0$ is necessary for stability but not sufficient since the growth coefficient obtained from the full expression (3.8) can be positive for some $q$ despite the coefficient $D$ being negative. In this sense, in general, the requirement $D<0$ will give only an upper bound for the stability region in $c_{1}, c_{2}$, and $k$ space. However, for the values of $c_{1}$ and $c_{2}$ considered in this work, the requirement $D<0$ gives a very good limit for the stability region.

For the unperturbed CGLE $(\gamma=0)$ the condition $D<0$ leads to the standard Eckhaus instability limit: $|k|<k_{E}$ with $k_{E}$ given by Eq. (2.3). For $\gamma \neq 0$ the first thing to notice is that independently of the value of the parameters $c_{1}$ and $c_{2}$, the added term never changes the stability of the homogeneous solution $k=0$. This can be seen from the fact that in Eqs. (3.6)-(3.8), $\gamma$ only appears in terms with powers of $k$. In general, the coefficient $D$ depends on even powers of $k$ up to the sixth power, so one has to solve a cubic equation to find explicitly the limits of the range of values of $k$ for which plane waves are stable. In Figs. 2-4 we plotted this range as a function of the parameter $c_{1}$ for several values of $\gamma$ and $c_{2}$.

Figure 2 shows the stability region for $c_{2}=-0.3$ and different values of $\gamma$ as indicated in the figure caption. For plane waves with $k \neq 0$ the stability range clearly changes with the value of $\gamma$ as displayed in Figs. 2(b)-2(d). For small 

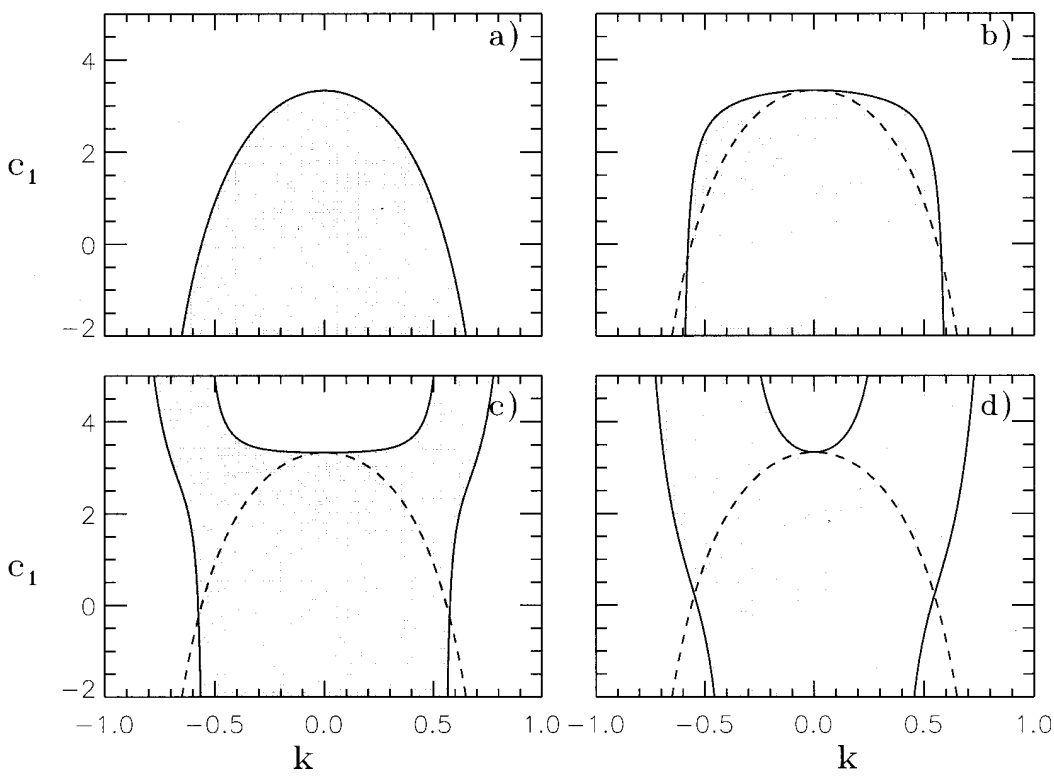

FIG. 2. Stability region for the plane wave (2.2) for $c_{2}=-0.3$ for (a) $\gamma=0$, (b) $\gamma=0.5$, (c) $\gamma=0.7$, and (d) $\gamma=2$. For comparison, the boundary of the stability region for $\gamma=0$ is shown in (b)-(d) as a dashed line.

$\gamma$ [Fig. 2(b)] the stability range is increased for large values of $c_{1}$ and slightly reduced for $c_{1} \lesssim-0.5$; therefore, the added stabilizing term has the opposite effect for small $c_{1}$. Increasing the value of $c_{1}$, the last plane wave in losing stability is still the homogeneous solution as it was in the case $\gamma=0$. For $\gamma>0.6$ the shape of the stability range is strongly changed, as can be seen in Figs. 2(c) and 2(d) for $\gamma=0.7$ and $\gamma=2$. Now there are plane waves with $k \neq 0$ that are stable for values of $c_{1}$ well above the BFN line, in the region of phase turbulence of the original CGLE (see Fig. 1).

Figures 3 and 4 show the stability regions for $c_{2}=-0.9$ and $c_{2}=-2.1$ and several values of $\gamma$. As $\gamma$ is increased the stability region changes its shape in a similar way to before but at larger values of $\gamma$. For $c_{2}=-0.9$ it is possible to stabilize plane waves in the region of phase turbulence tak- ing $\gamma \geqslant 2$, and for $c_{2}=-2.1$ stabilization in the region of defect turbulence is possible for $\gamma \geqslant 4$.

Figure 4(c) $\left(c_{2}=-2.1\right.$ and $\left.\gamma=4\right)$ shows an interesting intermediate shape. There are three stability regions, so plane waves can exist below the BFN line and well above it, in defect turbulence, but not for values of $c_{1}$ just above the $\mathrm{BFN}$ line. Also there is no wave vector $k$ for which plane waves are stable both below and above the BFN line. As $\gamma$ is increased the three regions coalesce and become a single one, as seen for $\gamma=6$. This is a general behavior also observed at other values of $c_{2}$ for intermediate values of $\gamma$ not shown in Figs. 2 and 3. The overall picture is as follows. For $\gamma=0$ and a fixed $c_{2}$ the stability region in the $k-c_{1}$ plane is limited by a branch of Eq. (2.3) (dashed line in Fig. 4) whose vertex corresponds to the BFN point. Decreasing the value of
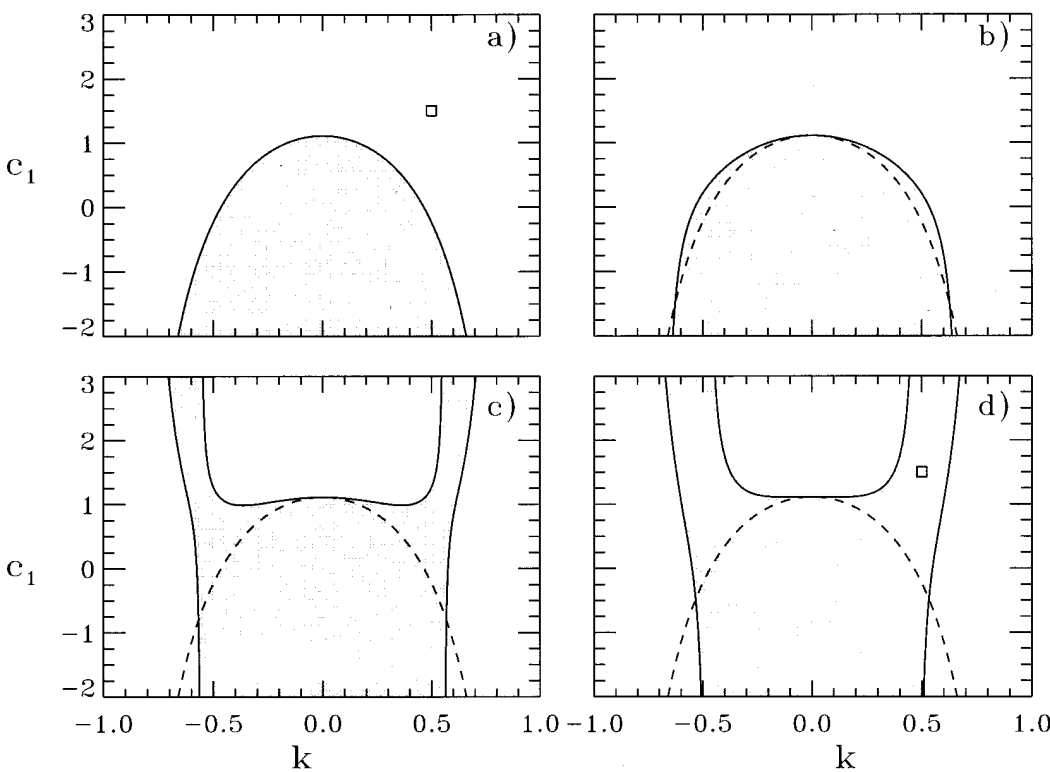

FIG. 3. Stability region for the plane wave (2.2) for $c_{2}=-0.9$ for (a) $\gamma=0$, (b) $\gamma=1$, (c) $\gamma=2$, and (d) $\gamma=3$. For comparison, the boundary of the stability region for $\gamma=0$ is shown in (b) $-(d)$ as a dashed line. 

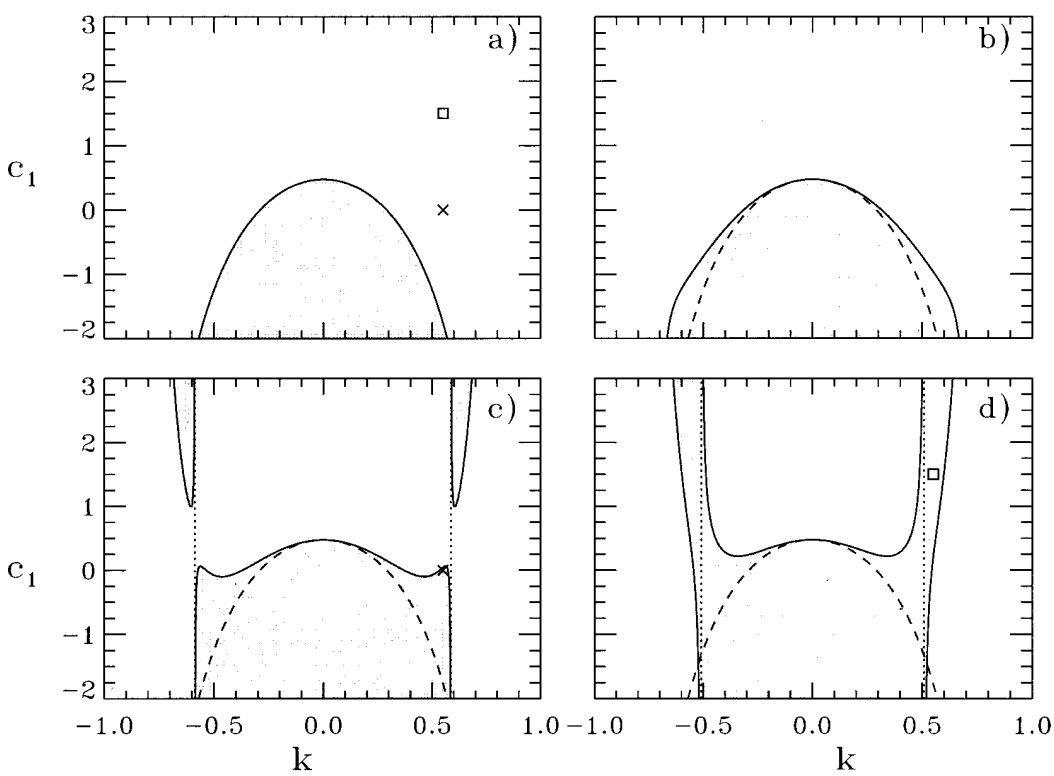

FIG. 4. Stability region for the plane wave (2.2) for $c_{2}=-2.1$ for (a) $\gamma=0$, (b) $\gamma=2$, (c) $\gamma=4$, and (d) $\gamma=6$. For comparison, the boundary of the stability region for $\gamma=0$ is shown in (b) $-(\mathrm{d})$ as a dashed line. The vertical dotted lines show the asymptotic lines $k=k_{A \pm}$ (see the text).

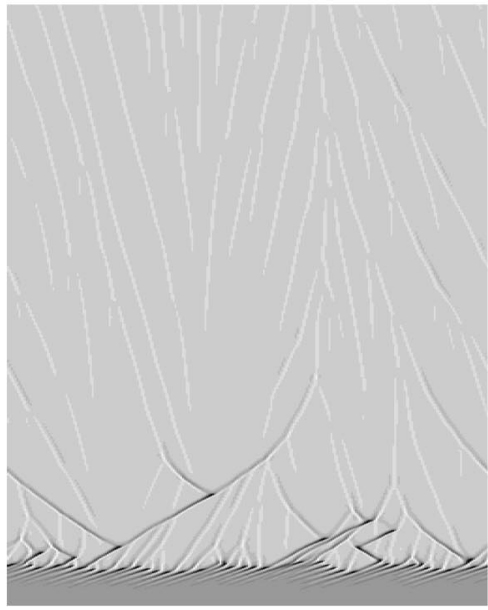

a)

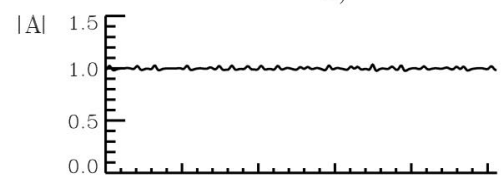
$\partial_{x} \varphi$

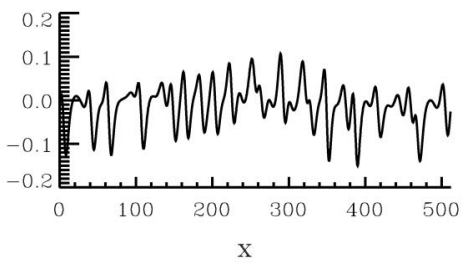

c)

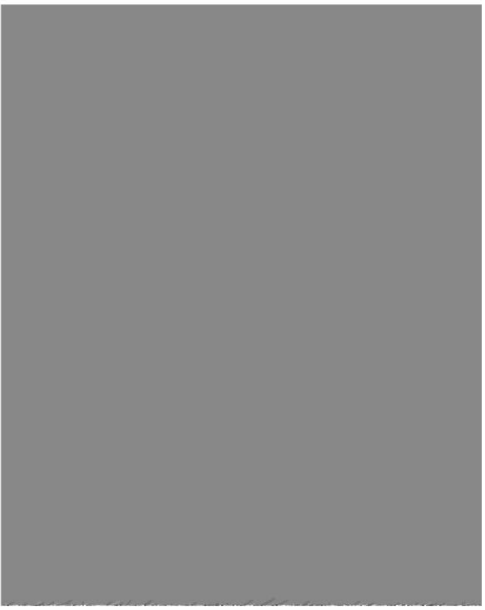

b)
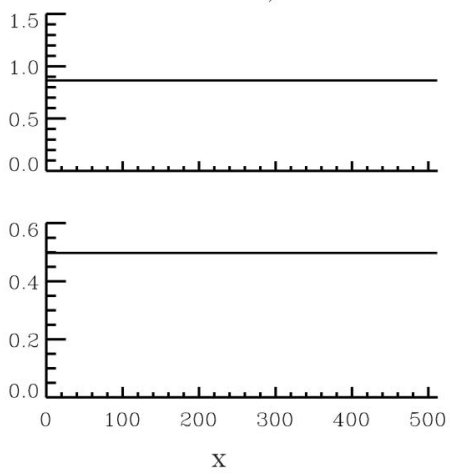

d)

FIG. 5. Spatiotemporal evolution of the CGLE (2.4) for $c_{1}=1.5$ and $c_{2}=-0.9$, starting from a perturbed plane wave (4.1) with $k=0.5$ and $\sigma=0.007$. (a) and (b) show $|A(x, t)|$ with time running upward from $t=0$ to $t=1000$ and $x$ in the horizontal direction for $\gamma=0$ and $\gamma=3$, respectively. The absolute value of the field $\left|A\left(x, t_{0}\right)\right|$ and the phase gradient $\partial_{x} \phi\left(x, t_{0}\right)$ at $t_{0}=950$ are displayed in (c) and (d) for $\gamma=0$ and $\gamma=3$, respectively. 


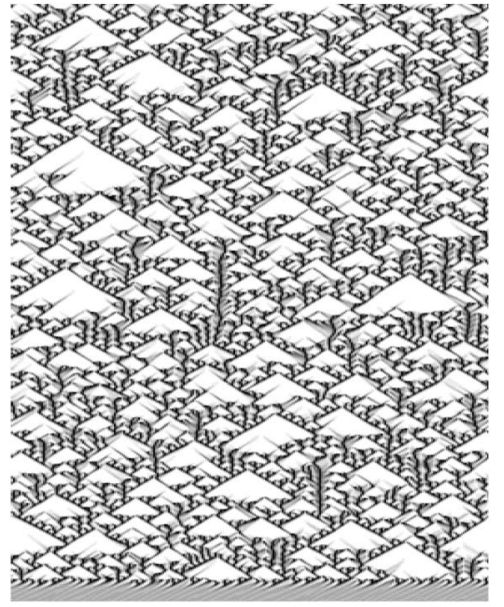

a)

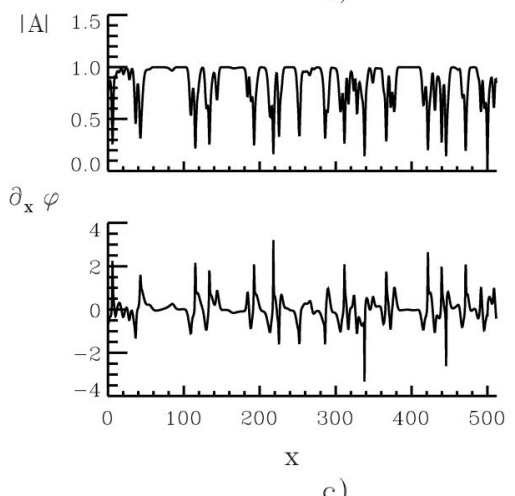

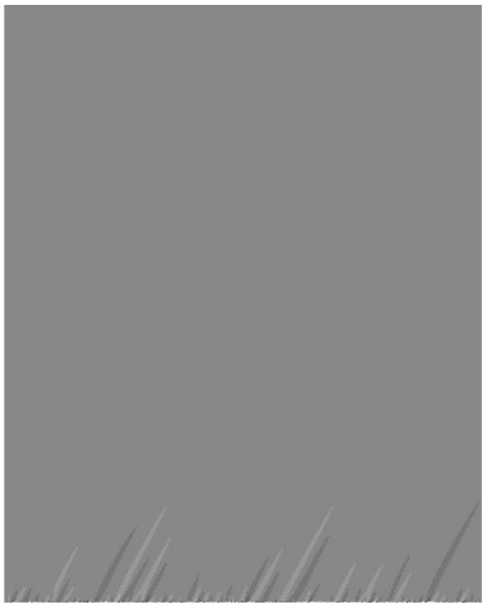

b)
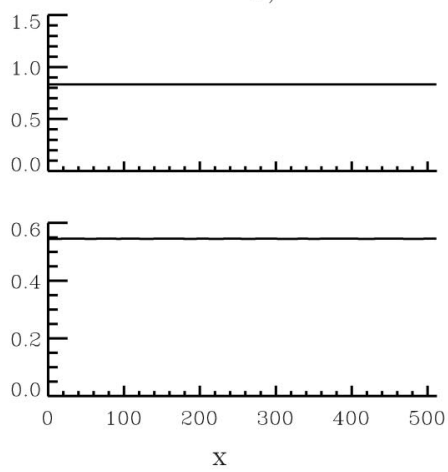

d)

FIG. 6. Spatiotemporal evolution of the CGLE for $c_{1}=0$ and $c_{2}=-2.1$, starting from a perturbed plane wave with $k=0.55$. (a) and (b) show $|A(x, t)|$ for $\gamma=0$ and $\gamma=4$, respectively. The values of $\left|A\left(x, t_{0}\right)\right|$ and $\partial_{x} \phi\left(x, t_{0}\right)$ at $t_{0}=950$ are displayed in (c) and (d) for $\gamma=0$ and $\gamma=4$, respectively. Other parameters are the same as in Fig. 5.

$c_{1}$, the width of the stability region $|k|<k_{E}$ increases and for $c_{1} \rightarrow-\infty, k_{E} \rightarrow 1$. For any small $\gamma>0$ there are three stability regions in the $k-c_{1}$ plane. From Eq. (3.10) one can show that for $c_{1} \rightarrow-\infty$ the limits of the central region approach the two vertical asymptotes $k_{A \pm}= \pm \sqrt{c_{2} /\left(c_{2}-\gamma\right)}$ [dotted lines in Figs. 4(c) and 4(d)]. Two new stability regions $(D<0)$ appear symmetrically at very large values of $c_{1}$ and for values of $|k|$ between the vertical asymptotes and 1 . The existence of these new regions, which broaden for $c_{1} \rightarrow \infty$ and cover the intervals $k \in\left[-1, k_{A-}\right]$ and $k \in\left[k_{A+}, 1\right]$, implies that for any nonvanishing $\gamma$ there will be always stable plane waves well above the BFN line. However, if $\gamma$ is very small these regions are located at very large, and quite unrealistic, values of $c_{1}$. As $\gamma$ is increased these regions extend to lower values of $c_{1}$ until they coalesce with the central region.

\section{NUMERICAL SIMULATIONS}

We have performed numerical simulations of Eqs. (2.1) and (2.4) using a pseudospectral code with periodic boundary conditions and second-order accuracy in time. Spatial resolution was typically 1024 modes. The time step was typically $\Delta t=0.001$. Since very small effects have been explored, care has been taken in confirming the invariance of the results with decreasing time step and increasing number of modes. The system size was always taken as $L=512$. The details of the numerical method can be seen in Ref. [28]. We start from an initial condition that corresponds to a plane wave plus a small random perturbation

$$
A(x, t=0)=\sqrt{1-k^{2}} e^{i k x}+\sigma \xi(x),
$$

where $\xi(x)$ is a complex Gaussian random perturbation of zero mean and variance $\left\langle\xi(x) \xi^{*}\left(x^{\prime}\right)\right\rangle=2 \delta\left(x-x^{\prime}\right)$.

We have performed numerical simulations in different regions of the phase diagram (Fig. 1) to verify the results obtained from the linear stability analysis when finite-size perturbations are applied. We have found a very good agreement between the prediction of the linear stability analysis and the numerical simulations. In the no chaos region we have tested the stabilization of plane waves with wave vector $|k|>k_{E}$ by using small values of $\gamma$ as predicted in Fig. 2 for $c_{1}>-0.5$. With $\gamma=0$, the perturbed unstable plane wave evolves towards another plane wave with wave vector $|k|<k_{E}$, whereas when the control term is added the initial perturbations are washed out by the dynamics and the system settles down to a plane wave with the initial wave vector. For $c_{1}<-0.5$, we have also tested cases where the added control term destabilizes an originally stable plane wave. In the modified CGLE, the initial plane wave evolves to another plane-wave solution with a smaller wave number that is inside the stability range given by the linear stability analysis. 


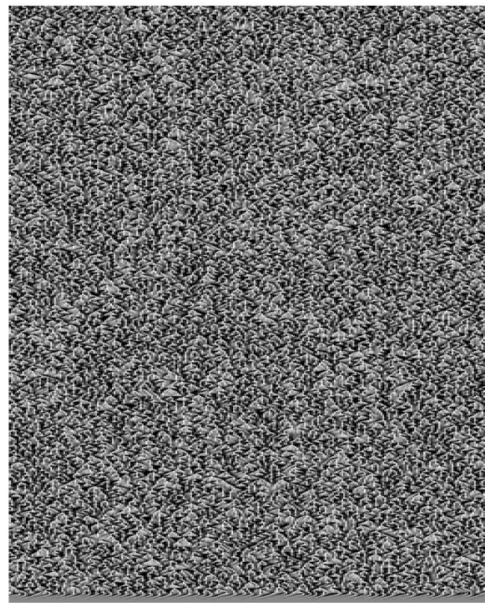

a)

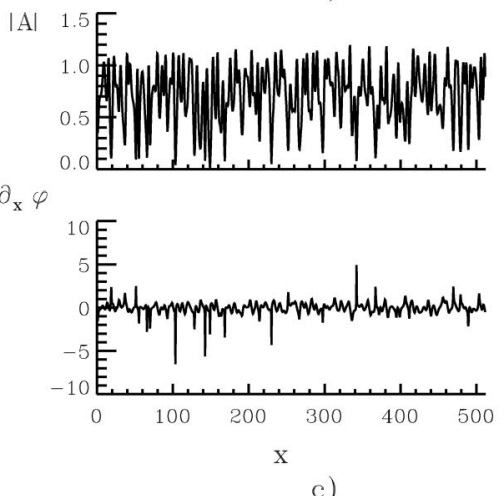

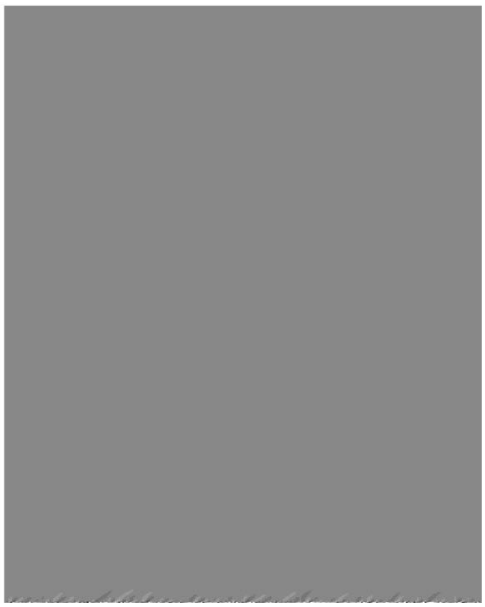

b)
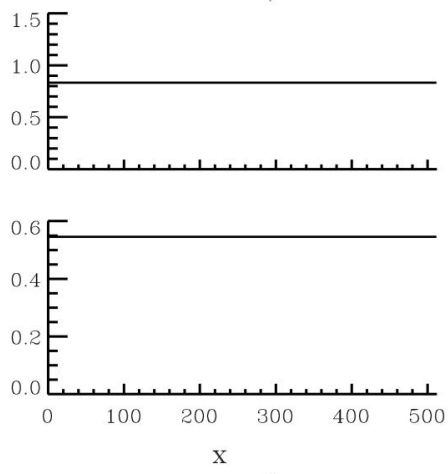

d)

FIG. 7. Spatiotemporal evolution of the CGLE for $c_{1}=1.5$ and $c_{2}=-2.1$, starting from a perturbed plane wave with $k=0.5$. (a) and (b) show $|A(x, t)|$ for $\gamma=0$ and $\gamma=6$, respectively. The values of $\left|A\left(x, t_{0}\right)\right|$ and $\partial_{x} \phi(x, t)$ at $t_{0}=950$ are displayed in (c) and (d) for $\gamma=0$ and $\gamma=6$, respectively. Other parameters are the same as in Fig. 5 .

Stabilization is also possible in the different regimes of STC found in the CGLE. As characteristic examples we show the following results.

Figure 5 shows the stabilization of a plane wave for parameter values $c_{1}=1.5$ and $c_{2}=-0.9$. This corresponds to the phase turbulence regime (see Fig. 1), where no plane waves are stable for the original CGLE. As predicted by the linear stability analysis [squares in Figs. 3(a) and 3(d)], a perturbed plane wave with $k=0.5$ can easily be stabilized with $\gamma=3$, while for $\gamma=0$ the same initial condition decays in time $t=80$ (approximately) to phase turbulence.

Figure 6 shows a case of stabilization of a plane wave for $c_{1}=0$ and $c_{2}=-2.1$, in the region of spatiotemporal intermittency. For the original CGLE, plane waves are stable in this region if $|k|$ is small enough, but if $|k|>k_{E}$ the initial perturbed plane wave evolves to a spatiotemporal intermittent behavior [7]. The nonlinear diffusion term proved to be an effective way of suppressing the evolution towards the disordered states (with defects and other localized structures), leading the system to a well-behaved plane wave. The initial condition in this case was a perturbed plane wave with $k=0.55$ [crosses in Figs. 4(a) and 4(c)].

Finally, Fig. 7, obtained for parameter values $c_{1}=1.5$ and $c_{2}=-2.1$, shows stabilization of plane waves in the region of defect turbulence, where for the unperturbed CGLE there are no stable plane waves and the field $A$ shows a strongly disordered STC state characterized by the presence of de- fects. As predicted by the linear stability analysis, a perturbed plane wave with $k=0.55$ [squares in Figs. 4(a) and 4(d)] can be stabilized with $\gamma=6$.

\section{CONCLUDING REMARKS}

We have stabilized unstable plane-wave solutions in different parameter regions of the CGLE where spatiotemporal chaos exists. This has been done by adding a term to the CGLE, which vanishes for the stabilized plane wave, so that the stabilized plane waves are exactly the same unstable solutions of the original CGLE. The added term can be seen as nonlinear diffusion and preserves the intrinsic phase invariance of the original equation. Although our method does not change the stability of the homogeneous solution $k=0$, it is quite effective in stabilizing plane waves with nonzero wave vectors. We have calculated analytically the parameter regions where plane waves can be stabilized, including regions of phase turbulence, spatiotemporal intermittency, bichaos, and defect turbulence. We have studied numerically the stability of the plane waves when finite-size perturbations are applied. The results are in excellent agreement with the analytical predictions of the linear stability analysis. Our analysis also shows that, in general, in systems where nonlinear diffusion or diffraction effects are not negligible, these terms can change substantially the regions in parameter space for which plane waves are stable. 
The basic purpose of this work was to show that stabilization of plane waves is possible by using a nonlinear diffusion term. The modified CGLE, however, can also have other kinds of solutions (chaotic or not) that are different from the ones of the original equation. From the analysis presented here one can draw some conclusions about the phase diagram of the modified CGLE. One is that the stability of the homogeneous solution is not changed, so it is stable only below the BFN line. Another is that for $\gamma$ large enough, there are always stable plane waves with wave vector $k \approx 0.5$, so the BFN line is no longer the limit of stability of plane waves. Numerically we have observed phase and defect turbulence regimes in the modified equation when starting from random initial conditions above the BFN line. However we have not done a systematic exploration for dif- ferent parameter values. A full phase diagram for the modified CGLE requires a very intensive numerical calculation, which is beyond the scope of this work.

\section{ACKNOWLEDGMENTS}

Financial support from DGICYT (Spain) Project Nos. PB94-1167 and PB94-1172 is acknowledged. R.M. also acknowledges partial support from the Programa de Desarrollo de Ciéncias Básicas (PEDECIBA, Uruguay), the Consejo Nacional de Investigaciones Científicas y Técnicas (CONICYT, Uruguay), and the Programa de Cooperación con Iberoamérica (ICI, Spain). The authors also acknowledge helpful discussions with M. San Miguel, E. HernándezGarcía, D. Walgraef, and G. Huyet.
[1] M. Cross and P. Hohenberg, Science 263, 1569 (1994).

[2] M. Dennin, G. Ahlers, and D. S. Cannell, Science 272, 388 (1996).

[3] M. Cross and P. Hohenberg, Rev. Mod. Phys. 65, 851 (1993), and references therein.

[4] W. van Saarloos and P. Hohenberg, Physica D 56, 303 (1992); 69, 209(E) (1993).

[5] H. Chate, in Spatiotemporal Patterns in Nonequilibrium Complex Systems, Vol. XXI of Santa Fe Institute in the Sciences of Complexity, edited by P. Cladis and P. Palffy-Muhoray (Addison-Wesley, New York, 1995), pp. 5-49.

[6] B. Shraiman et al., Physica D 57, 241 (1992).

[7] H. Chaté, Nonlinearity 7, 185 (1994).

[8] D. Egolf and H. Greenside, Phys. Rev. Lett. 74, 1751 (1995).

[9] R. Montagne, E. Hernández-García, and M. San Miguel, Physica D 96, 47 (1996).

[10] R. Montagne, E. Hernández-García, and M. San Miguel, Phys. Rev. Lett. 77, 267 (1996).

[11] T. Shinbrot, C. Grebogi, E. Ott, and J. Yorke, Nature (London) 363, 411 (1993).

[12] I. Aranson, H. Levine, and L. Tsimring, Phys. Rev. Lett. 72, 2561 (1994).

[13] M. Bleich and J. E. S. Socolar, Phys. Lett. A 210, 87 (1996).

[14] F. Mertens, R. Imbihl, and A. Mikhailov, J. Chem. Phys. 101, 9903 (1994).
[15] D. Battogtokh and A. Mikhailov, Physica D 90, 84 (1996).

[16] H. Gang, Phys. Rev. Lett. 71, 3794 (1993).

[17] W. Lu, D. Yu, and R. Harrison, Phys. Rev. Lett. 76, 3316 (1996).

[18] D. Hochheiser, J. V. Moloney, and J. Lega (unpublished); M. E. Bleich, D. Hochheiser, J. V. Moloney, and J. E. S. Socolar, Phys. Rev. E 55, 2119 (1997).

[19] W. van Saarloos, in Spatiotemporal Patterns in Nonequilibrium Complex Systems (Ref. [5]), p. 19.

[20] A. C. Newell, T. Passot, and J. Lega, Annu. Rev. Fluid Mech. 25, 399 (1993).

[21] W. Eckhaus, Studies in Nonlinear Stability Theory (Springer, Berlin, 1965).

[22] A. Torcini, Phys. Rev. Lett. 77, 1047 (1996).

[23] H. Chaté and P. Manneville, Physica A 224, 348 (1996).

[24] H. Chaté and P. Manneville, in A Tentative Dictionary of Turbulence, edited by $\mathrm{P}$. Tabeling and $\mathrm{O}$. Cardoso (Plenum, New York, 1995).

[25] P. Manneville and H. Chaté, Physica D 96, 30 (1996).

[26] J. Lega, Ph.D. thesis, Université de Nice, 1989 (unpublished).

[27] B. Janiaud et al., Physica D 55, 269 (1992).

[28] R. Montagne, E. Hernández-García, A. Amengual, and M. San Miguel, Phys. Rev. E 56, 151 (1997). 